\title{
Biochemical heterozygosity and phenotypic variability of polygenic traits
}

\author{
Ranajit Chakraborty
}

\author{
Center for Demographic and Population Genetics, \\ University of Texas Graduate School of Biomedical \\ Sciences, P.O. Box 20334, Houston, Texas 77225, \\ U.S.A.
}

Using the theory of additive genetic variability of a polygenic trait, it is shown than an individual's heterozygosity at the loci governing the trait cannot be determined accurately from observations on phenotypes alone. Furthermore, the negative association between heterozygosity and phenotypic variance, and a positive correlation of the frequency of the modal class of a phenotypic trait and the extent of heterozygosity can be explained by additive allelic effects. It is argued that while the number of heterozygous loci in an individual may not be a good indicator of the individual's genomic heterozygosity, there is evidence that some of the biochemical loci may reflect genetic variation at the loci controlling phenotypic polymorphism. Thus the observed relationship between biochemical heterozygosity and phenotypic variance may not constitute hard evidence of heterosis, overdominance, or associative overdominance.

\section{INTRODUCTION}

Allozymic variations in natural populations, studied over the past two decades, have now convincingly established that genetic variation is generally ubiquitous in almost all taxa. Recent molecular data showing genic variations at the DNA level have strengthened this view (Nei, 1987). Yet, the controversy regarding the mechanistic explanations for the production and maintainance of genetic polymorphism is still unresolved, since direct searches for selection at any individual locus have produced ambiguous results at best. More recently, therefore, attempts have been made to relate the heterozygosity levels detected by multiple loci with variabilities in morphological or fitness related traits in order to test heterotic effects of the loci studied. Critical appraisals of the use of allozyme data in this regard have been made in recent reviews of Mitton and Grant (1984) and Zouros and Foltz (1987). These reviews and their cited references indicate that the efforts to relate allozymic heterozygosity with phenotypic variability do not always produce positive results. Yet, in many organisms, from plants to human, associations between these two parameters suggest that heterotic effects may be detectable by studying interactions of multiple loci together. The concept of developmental homeostasis has also been invoked to explain the observation of positive associations between the heterozygosity and the lack of phenotypic variability (see e.g., the references cited in the above two reviews, and Livshits and Kobyliansky, 1985).

The main features of the positive results of such association studies may be summarised into three observations. (a) When heterozygosity is measured by the number of loci at which an individual is heterozygous, increased heterozygosity is often associated with a decreased phenotypic variability. (b) The frequency of modal phenotypes is positively correlated with the heterozygosity levels of individuals. (c) Parental fitness is positively associated with the expected degree of heterozygosity among offspring. None of these findings are, however, universal, since exceptions are found in repeated studies in the same population (Pierce and Mitton, 1982), and in studies involving populations of different evolutionary origin (e.g., Gottlieb, 1977; Handford, 1980; Knowles and Mitton, 1980; Knowles and Grant, 1981; Mitton et al., 1981; Chakraborty et al., 1986).

In spite of these discordant results, the above features of association of enzymic heterozygosity with phenotypic variability demand satisfactory mechanistic explanation. The interpretation of selective differentials among individuals involve the assumption that the morphological traits used 
in determining phenotypic variability are genetically controlled, and the biochemical loci employed reflect genetic variation at the loci controlling such traits. It is true that genetic factors are involved in morphological variation, and fitness may be genetically controlled. Yet, the role of non-genetic modifiers in determining phenotypes of such traits cannot be totally ignored. Furthermore, the task is more complicated, since the number and nature of genetic loci involved in morphological or fitness related traits are not known, and their relationships with structural biochemical loci are not clear.

The purpose of this paper is to show that the classification of individuals into different heterozygosity classes by phenotypes of a polygenic trait is quite error prone, even if the trait is under complete genetic control. It is also shown that the increased frequency of modal phenotypes in highly heterozygous individuals (and the consequent decrease of phenotypic variability in them) is a direct consequence of additivity of allelic effects of a polygenic trait. Lastly, the question of interdependence of heterozygosity at biochemical and phenotypic level is addressed by reviewing the theory of predictability of genomic heterozygosity using electrophoretic markers, in view of the recent comments of Zouros and Foltz (1987) and Smouse (1986).

\section{DISTRIBUTION OF HETEROZYGOSITY BY PHENOTYPE CLASSES}

In relation to the studies of morphological variation and the heterozygous nature of individuals, it is common to identify the heterozygous status of individuals by their phenotypic scores for a heritable polygenic trait (see e.g., Livshits and Kobyliansky, 1984; 1985; Kobyliansky and Livshits, 1985). This is based on the supposition that the phenotypic value of a morphological trait is determined by polygenic effects of the loci controlling the trait, and the allelic effects are additive. Nevertheless, there has been no attempt to check this analytically. To address this question, let us consider a quantitative trait controlled by $n$ loci, at each of which there are two segregating alleles (say, $A_{i}$ and $B_{i}$ for the $i$ th locus; $i=$ $1,2, \ldots, n)$. For simplicity, let us assume that the allelic effects are all additive. Without loss of generality we can assume that the allelic affects of $A_{i}$ is one and the effect of $B_{i}$ is zero, for all $i=$ $1,2, \ldots, n$. We shall further assume that the allele frequencies at the $i$ th locus are $p_{i}$ (for the allele
$\left.A_{i}\right)$ and $q_{i}\left(\right.$ for the allele $B_{i}$ ), where $q_{i}=1-p_{i}$. The heterozygosity for each individual is measured by the number of heterozygous loci, if there are genotypic scoring techniques available for these loci. Let $Y$ denote the number of heterozygous loci in a random individual, and $X$ denote the genotypic value of the individual. We can write $X$ and $Y$ as

$$
X=X_{1}+X_{2}+\cdots+X_{n}
$$

and

$$
Y=Y_{1}+Y_{2}+\cdots+Y_{n},
$$

where the bivariate distribution of $X_{i}$ and $Y_{i}$ is given by

\begin{tabular}{llll}
\hline$X_{i}$ & $Y_{i}$ & Genotype & Probability \\
\hline 2 & 0 & $A_{i} A_{i}$ & $p_{i}^{2}$ \\
1 & 1 & $A_{i} B_{i}$ & $2 p_{i} q_{i}$ \\
0 & 0 & $B_{i} B_{i}$ & $q_{i}^{2}$ \\
\hline
\end{tabular}

Under the assumption that the loci are independently segregating in the population, the joint distribution of $(X, Y)$ can be evaluated by the bivariate probability generating function method (Feller, $1950)$; i.e., the probability that $X=r$ and $Y=k$ is given by

$$
\begin{aligned}
\operatorname{Prob}(X & =r, Y=k) \\
& =\text { Coefficient of } s_{1}^{r} s_{2}^{k} \text { in } G\left(s_{1}, s_{2}\right),
\end{aligned}
$$

where

$$
G\left(s_{1}, s_{2}\right)=\prod_{i=1}^{n}\left(q_{i}^{2}+2 p_{i} q_{i} s_{1} s_{2}+p_{i}^{2} s_{1}^{2}\right),
$$

for $r=0,1,2, \ldots, 2 n ; k=0,1,2, \ldots, n$, and $s_{1}, s_{2}$ are two arbitrary variables taking values between -1 and 1.

Note that the marginal distributions of $X$ and $Y$ can be obtained by evaluating the coefficients of $s_{1}^{r}$ in $G\left(s_{1}, 1\right)$ and $s_{2}^{k}$ in $G\left(1, s_{2}\right)$, respectively. Chakraborty (1981) provided a computational algorithm for computing the distribution of $Y$, and the same technique also applies to $X$. Since for the quantitative phenotypes, the distribution of $Y$ cannot be generally observed directly, one might be interested in drawing inference regarding it by the conditional distribution of $Y$ (number of heterozygous loci) given an observation on $X$ (the phenotypic score). In practice, however, this is complicated by the effects of non-genetic environmental factors which may modify the genotypic score $X$. For simplicity, if we assume that $X$ is 
directly observable and is unaffected by environmental factors, the conditional distribution of $Y$ given $X=r$ is given by

$$
\text { Prob } \begin{aligned}
(Y & =k \mid X=r) \\
& =\frac{\text { Coefficient of } s_{1}^{r} s_{2}^{k} \text { in } G\left(s_{1}, s_{2}\right)}{\text { Coefficient of } s_{1}^{r} \text { in } G\left(s_{1}, 1\right)},
\end{aligned}
$$

for $0 \leqq r \leqq 2 n$, and $0 \leqq k \leqq n$.

For the case where the gene frequencies at all loci are equal, i.e., $p_{i}=p$ for all $i$, it is easy to show that

$$
\operatorname{Prob}(X=r)=\left(\begin{array}{c}
2 n \\
r
\end{array}\right) p^{r} q^{2 n-r}
$$

and

$$
\operatorname{Prob}(Y=k)=\left(\begin{array}{l}
n \\
k
\end{array}\right)(2 p q)^{k}\left(p^{2}+q^{2}\right)^{n-k} .
$$

Furthermore, in this case the multi-locus genotype of any individual can be represented by a triplet $\left(n_{1}, n_{2}, n_{3}\right)$, where $n_{1}, n_{2}$, and $n_{3}$ represent the numbers of loci exhibiting genotypes $A A, A B$, and $B B$, respectively; with $n_{1}+n_{2}+n_{3}=n$. Thus, the conditional probability of equation becomes

$$
\operatorname{Prob}(Y=k \mid X=r)=\frac{\operatorname{Prob}\left(2 n_{1}+n_{2}=r, n_{2}=k\right)}{\operatorname{Prob}\left(2 n_{1}+n_{2}=r\right)},
$$

which may be written as

$$
\begin{aligned}
\operatorname{Prob}( & Y=k \mid X=r) \\
= & \frac{\operatorname{Prob}\left[n_{1}=\frac{r-k}{2}, n_{2}=k, n_{3}=n-\frac{r+k}{2}\right]}{\operatorname{Prob}\left(2 n_{1}+n_{2}=r\right)} \\
= & \left(\frac{n !}{\{(r-k) / 2\} ! k !\{n-(r+k) / 2\} !}\right. \\
& \left.\times p^{(r-k)}(2 p q)^{k} q^{2 n-(r+k)}\right) \\
& /\left(\frac{(2 n) !}{r !(2 n-r) !} p^{r} q^{2 n-r}\right) \\
= & \frac{B(n, 1 / 2 ; k) B\left(n-k, 1 / 2 ; \frac{r-k}{2}\right)}{B(2 n, 1 / 2 ; r)},
\end{aligned}
$$

where $B(n, p ; m)$ denotes the binomial probability for $m(0 \leqq m \leqq n)$ with parameters $n$ and $p$.
It is interesting to note that when all loci controlling the quantitative trait $X$ have the same allele frequencies, the conditional distribution of $Y$ given $X$ does not depend on the allele frequency $p$. Furthermore, since equation (6) is defined only for even values of $r-k$, for a fixed value of $r(0 \leqq$ $r \leqq 2 n), k$ can take values from 0 to min. $(r, 2 n-r)$, such that $r-k$ is always even. Equations (3) and (6) are instructive enough to show that the classification of individuals into heterozygosity classes by phenotypic scores can be quite misleading. This is numerically demonstrated in table 1 , where the conditional distribution of $Y$ (the number of heterozygous loci) for different genotypic values $(X)$ are shown for the case where the allele frequencies are identical for all loci (the exact value is irrelevant, since eqn. (6) is independent of $p$ ).

Two situations are illustrated with table 1 , the number of loci $(n)$ being 5 and 10 . In both cases it is seen that the central phenotype $(X=5$ and $10)$ can have heterozygosity at less than half of the loci with probability approaching $1 / 4$ or more. It can be shown from eqn. (3) or (6), that the mean number of heterozygous loci increases gradually from extreme to central phenotypes, numerical illustrations of which are given in fig. 1. However, since the distributions of the number of heterozygous loci are overlapping for different phenotypic classes (see table 1), classification of individuals into different heterozygous classes by phenotypes of individuals can be quite error prone.

It is also interesting to note that while the conditional distribution of the number of heterozygous loci given the phenotypic score is independent of the allele frequency $(p)$, when all loci have identical allele frequencies, the highest number of heterozygous loci may not always occur for modal phenotypes. For example, if the allele frequencies are skewed (i.e., $p<0.5$ or $p>0.5$ ), the modal genotypic score will not be exactly at the middle of the range of $X$, while the average number of heterozygous loci will always be highest for the central value of $X$. This is graphically shown in fig. 1 , where the distribution of $X$ is given for $n=10$, for three values of $p(0 \cdot 3,0 \cdot 5$, and $0 \cdot 7)$ by the solid lines, and the dotted lines show the mean \pm sd of the number of heterozygous loci for each value of $X$ for the same value of $n$ (the means are represented by closed circles). These clearly demonstrate that the number of heterozygous loci may not be always associated with the modal phenotypic score, and hence the predictability of heterozygous status of individuals by phenotypic scores alone can be misleading. 
Table 1 Conditional distribution of the number of heterozygous loci $(Y)$ given genotypic value $(X)$

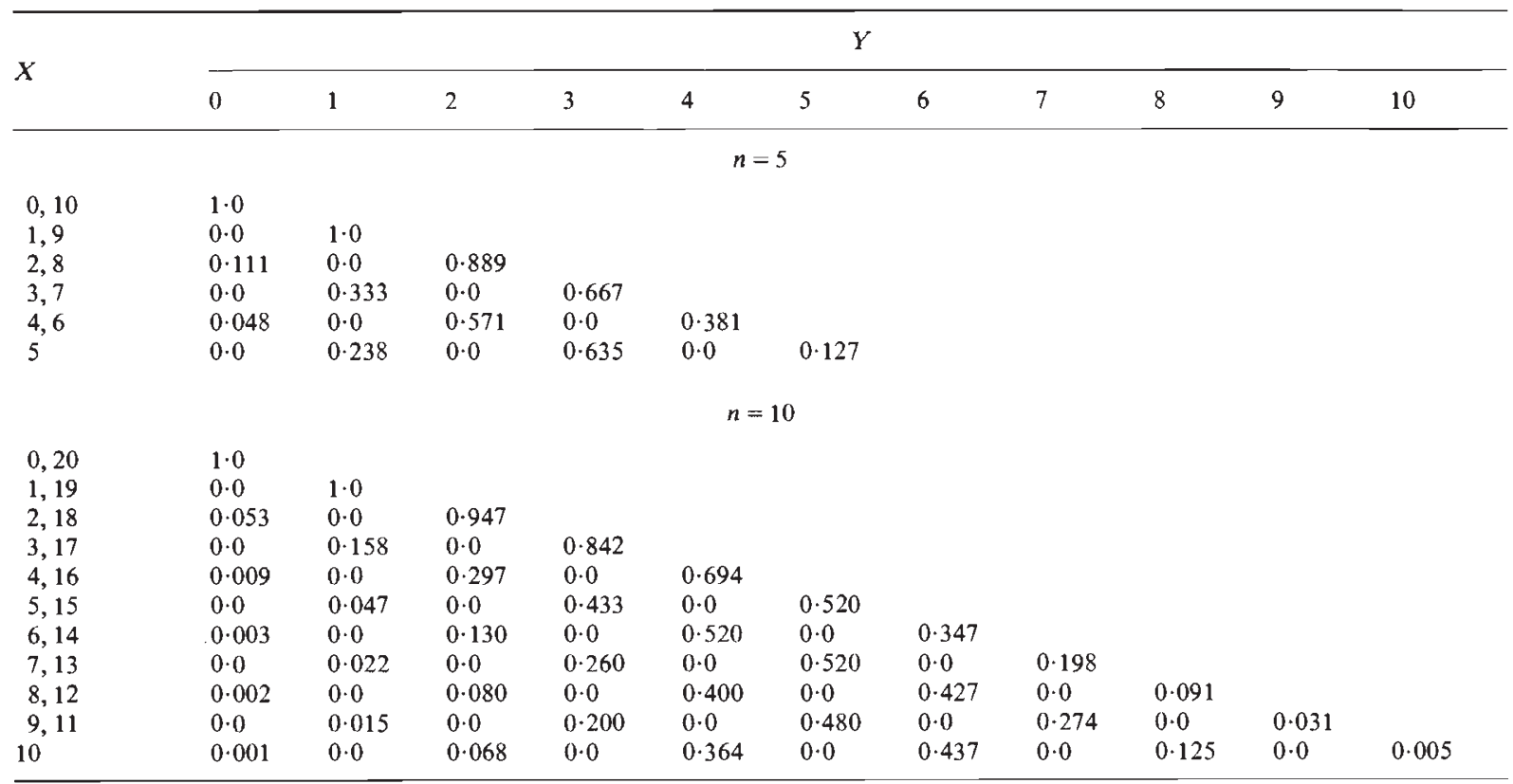

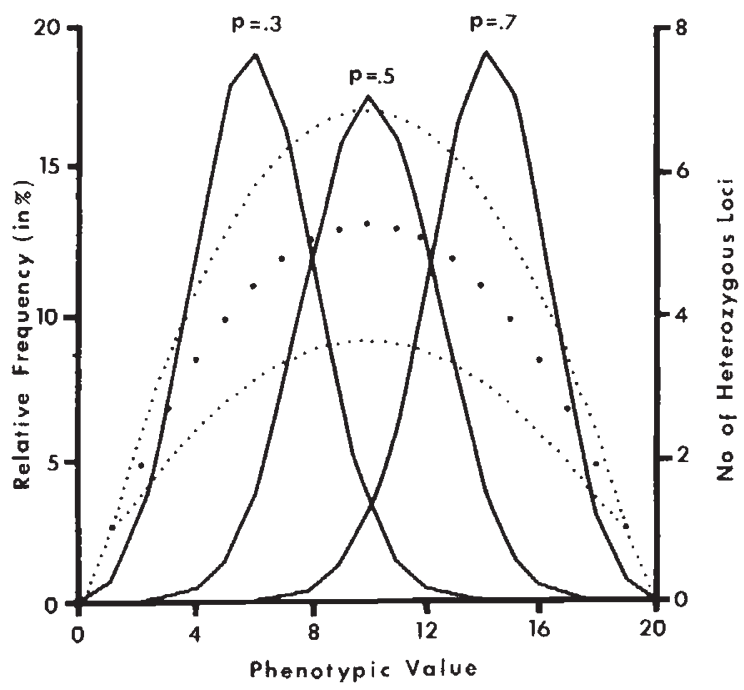

Figure 1 Relationship between the mean number of heterozygous loci underlying a polygenic quantitative trait given the phenotypic score and the distribution of the trait controlled by 10 loci. The solid lines give the distributions of phenotypic scores in the population (for gene frequencies $p=0.3,0.5$, and 0.7 ), and the dotted lines represent mean \pm s.d. of the number of heterozygous loci given the phenotypic scores. The closed circles represent the mean values of number of hetrozygous loci given phenotypic values (see text for details).
In analysis of data of this type, individuals are often classified into modal type and extreme type by grouping them into classes by phenotypes (e.g., Livshits and Kobyliansky, 1984; 1985). For example, if the individuals are classified into three classes: $E_{0} \quad(X \leqq$ mean $-0.67 \mathrm{sd}), \quad M$ (mean$0.67 \mathrm{sd}<X \leqq$ mean $+0.67 \mathrm{sd}) ;$ and $E_{1} \quad(X>$ mean $+0.67 \mathrm{sd})$, it is not always true that the heterozygosity level will be highest for the $M$ class. Table 2 provides numerical calculations of such values from the theoretical distributions presented above. For numerical illustrations, we have chosen three values of $p(0.3,0.5$, and 0.7$)$ and two values of $n$ ( 5 and 10) for each of which the three classes ( $E_{0}, M$, and $E_{1}$ ) were defined by the corresponding mean and sd of the phenotypic scores $(X)$, and within each class the distribution of number of heterozygous loci is computed. As a summary measure, table 2 shows only the mean and sd of the number of heterozygous loci within each class. These calculations demonstrate that the phenotypic class where the maximum average heterozygosity occurs is dependent on the allele frequencies, and the central class $(M)$ is not always the one which exhibits the highest average heterozygosity. 
Table 2 Mean and standard deviation of the number of heterozygous loci for different phenotypic classes of a polygenic trait

\begin{tabular}{|c|c|c|c|c|c|}
\hline \multirow{2}{*}{$n$} & \multirow{2}{*}{$p$} & \multicolumn{2}{|c|}{ Phenotype } & \multicolumn{2}{|c|}{$\begin{array}{l}\text { No. of heterozygous } \\
\text { loci }\end{array}$} \\
\hline & & class & prob. & mean & s.d. \\
\hline \multirow[t]{3}{*}{5} & $0 \cdot 3$ & $E_{0}$ & $0 \cdot 383$ & 1.401 & 0.723 \\
\hline & & $M$ & $0 \cdot 267$ & $2 \cdot 333$ & 0.942 \\
\hline & & $E_{1}$ & $0 \cdot 350$ & $2 \cdot 686$ & $1 \cdot 144$ \\
\hline \multirow[t]{3}{*}{5} & 0.5 & $E_{0}$ & $0 \cdot 172$ & $2 \cdot 102$ & 0.930 \\
\hline & & $M$ & 0.656 & $2 \cdot 708$ & $1 \cdot 151$ \\
\hline & & $E_{1}$ & $0 \cdot 172$ & $2 \cdot 102$ & 0.930 \\
\hline \multirow[t]{3}{*}{5} & 0.7 & $E_{0}$ & $0 \cdot 350$ & $2 \cdot 686$ & $1 \cdot 144$ \\
\hline & & $M$ & $0 \cdot 267$ & $2 \cdot 333$ & 0.942 \\
\hline & & $E_{1}$ & $0 \cdot 383$ & $1 \cdot 401$ & 0.723 \\
\hline \multirow[t]{3}{*}{10} & 0.3 & $E_{0}$ & 0.237 & $2 \cdot 910$ & $1 \cdot 041$ \\
\hline & & $M$ & 0.535 & $4 \cdot 376$ & $1 \cdot 366$ \\
\hline & & $E_{1}$ & $0 \cdot 228$ & $5 \cdot 133$ & 1.583 \\
\hline \multirow[t]{3}{*}{10} & 0.5 & $E_{0}$ & 0.252 & $4 \cdot 774$ & $1 \cdot 515$ \\
\hline & & $M$ & 0.496 & $5 \cdot 229$ & $1 \cdot 613$ \\
\hline & & $E_{1}$ & $0 \cdot 252$ & $4 \cdot 774$ & $1 \cdot 515$ \\
\hline \multirow[t]{3}{*}{10} & 0.7 & $E_{0}$ & 0.228 & $5 \cdot 133$ & $1 \cdot 583$ \\
\hline & & $M$ & 0.535 & $4 \cdot 376$ & $1 \cdot 366$ \\
\hline & & $E_{1}$ & 0.237 & $2 \cdot 910$ & $1 \cdot 041$ \\
\hline
\end{tabular}

Note: the classes are defined as $E_{0}: X \leqq \mu-0.67 \sigma$; $M: \mu-0.67 \sigma<X \leqq \mu+0.67 \sigma$; and $E_{1}: X>\mu+0.67 \sigma$; where $\mu=2 n p$, and $\sigma=\sqrt{2 n p q}$.

DISTRIBUTION OF PHENOTYPIC SCORES FOR GIVEN HETEROZYGOSITY

The theory presented in the previous section shows that when the loci that control a polygenic trait are not known, the level of heterozygosity at these loci cannot always be accurately predicted from the phenotypic values alone. One could, however, ask a related but somewhat different question. If such loci can be scored, to what extent is the phenotypic variability associated with the individual's heterozygosity? Chakraborty and Ryman (1983) conducted a theoretical study of this problem, and demonstrated that the phenotypic variability is the least for individuals that are heterozygous for most loci. In other words, the maximum variance of $X$ is expected to occur for individuals who have $Y$ values at the extreme (i.e., $Y=0$ or $n$ ). Zouros and Foltz (1987) claim that the frequency of such individuals is very small in the population, since most individuals will demonstrate heterozygosity of intermediate levels. This, however, does not negate the fact that polygenic traits with additive allelic effects always produce a negative correlation between heterozygosity (as determined by number of heterozygous loci) and phenotypic variance, and for this to happen there is no need to invoke a selection hypothesis for the trait or for the underlying loci. Using the formulation of the previous section, we can evaluate the distribution of phenotypic scores for a given number of heterozygous loci.

In general, the probability that $X=r$ given $Y=k$ can be written as

$$
\text { Prob } \begin{aligned}
(X & =r \mid Y=k) \\
& =\frac{\text { Coefficient of } s_{1}^{r} s_{2}^{k} \text { in } G\left(s_{1}, s_{2}\right)}{\text { Coefficient of } s_{2}^{k} \text { in } G\left(1, s_{2}\right)},
\end{aligned}
$$

where $G\left(s_{1}, s_{2}\right)$ is as defined in equation (2).

In particular, when allele frequencies are equal at all loci,

$$
\operatorname{Prob}(X=r \mid Y=k)=\frac{\operatorname{Prob}\left(2 n_{1}+n_{2}=r, n_{2}=k\right)}{\operatorname{Prob}\left(n_{2}=k\right)},
$$

where $n_{1}, n_{2}$ are as defined earlier.

Algebraic simplication yields the equation

$$
\begin{aligned}
\operatorname{Prob}(X= & r \mid Y=k)=\left(\begin{array}{c}
n-k \\
\{r-k\} / 2
\end{array}\right) \\
& \times\left[\frac{p^{2}}{p^{2}+q^{2}}\right]^{\{r-k\} / 2}\left[\frac{q^{2}}{p^{2}-q^{2}}\right]^{(n-k)-\{r-k\} / 2}
\end{aligned}
$$

defined for even values of $r-k$, i.e., for $r=k, k+2$, $k+4, \ldots \min (2 n-k)$.

This shows that the mean and variance of phenotypic scores for a given level of heterozygosity $(Y=k)$ are

$$
\mu_{k}=\left[2 n p^{2}+k\left(q^{2}-p^{2}\right)\right] /\left(p^{2}+q^{2}\right),
$$

and

$$
\sigma_{k}^{2}=4(n-k) p^{2} q^{2} /\left(p^{2}+q^{2}\right)^{2},
$$

respectively. Note that equations (10) and (11) are identical to equations (4) and (5) of Chakraborty and Ryman (1983).

Figure 2 illustrates the dependence of the mean $\left(\mu_{k}\right)$ and s.d. $\left(\sigma_{k}\right)$ of phenotypic scores on the number of heterozygous loci, for $n=10$, for three specific values of $p(0 \cdot 3,0 \cdot 5$, and $0 \cdot 7)$. Two implications of this theory are noteworthy in relation to the studies of phenotypic variability and heterozygosity: (a) it is clear that irrespective of allele frequencies at the loci governing the trait, phenotypic variance is a decreasing function of the number of heterozygous loci; and (b) as a consequence of the fact that the variance $\left(\sigma_{k}^{2}\right)$ 


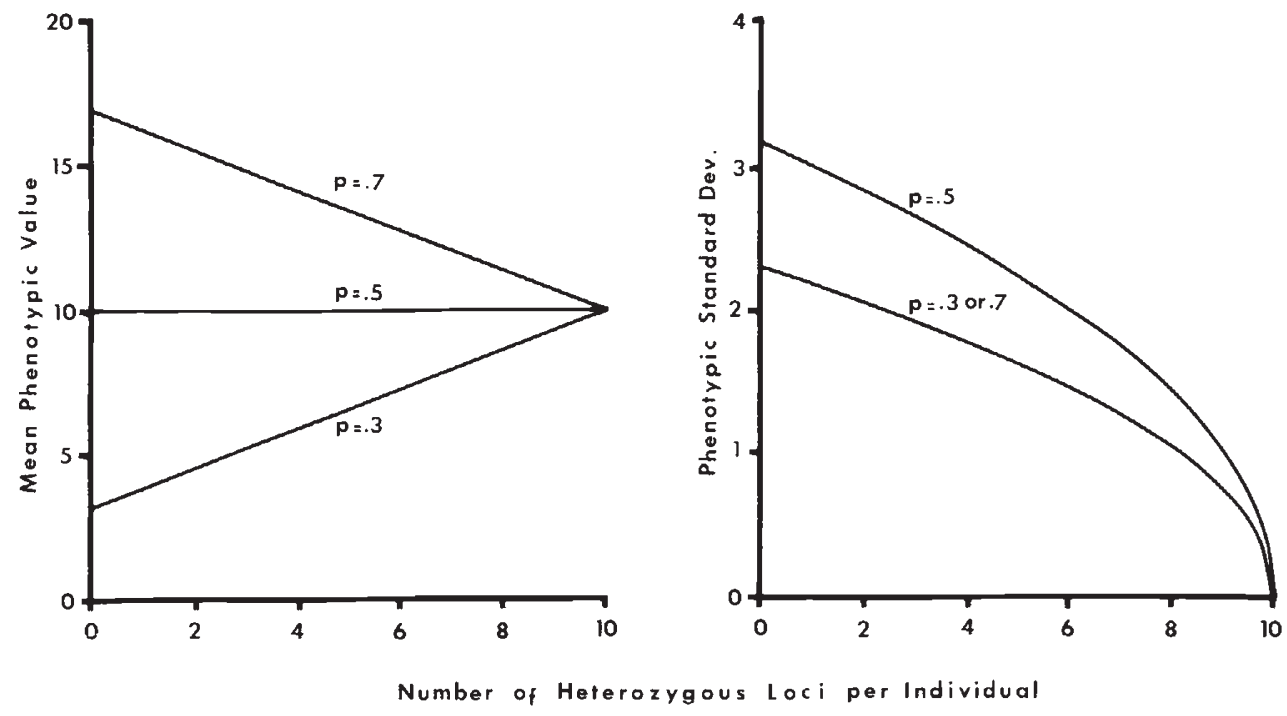

Figure 2 Relationship of the number of heterozygous loci with (a) mean and (b) standard deviation of the phenotypic score of a polygenic trait with complete heritability. For these computations the trait is assumed to be controlled by 10 loci at each of which the allele frequencies of two alleles are the same (p).

decreases with $k$ and the mean $\left(\mu_{k}\right)$ approaches the central value as $k$ increases, the frequency of central classes of phenotypes increases as the individuals exhibit more heterozygosity. Therefore, we conclude that when heterozygosity of individuals reflect heterozygosity at the loci governing a quantitative trait, the above two observations are direct consequences of the additive allelic effects of the loci, and no selective differential is necessary to explain these findings.

\section{HETEROZYGOSITY AT BIOCHEMICAL LOCI AS A REFLECTION OF GENOMIC HETEROZYGOSITY}

In relation to the studies of heterozygosity and variability of morphological or fitness related traits, commonly only one to about a dozen biochemical loci are used (see e.g., Zouros and Foltz, 1987 and the cited references in that review). If these loci are not directly involved in determining the trait in question, one might ask how well does the individuals' heterozygosity detected by these loci reflect the genomic heterozygosity of the individual? Mitton and Pierce (1980) and Chakraborty (1981) addressed this problem. Under the assumption that if $L$ loci from a collection of $N$ loci are randomly sampled, Chakraborty's analytical treatment shows that the correlation between the heterozygosity measured by $L$ loci and the heterozygosity for all $N$ loci is roughly equal to $(L / N)^{1 / 2}$, although the exact value is dependent on the mean and variance in heterozygosity values over $N$ loci (Chakraborty, 1981). Since the fraction, $L / N$, in all empirical studies is very small, Chakraborty asserted that the heterozygosity of an individual determined by the traditional biochemical markers does not provide an accurate indicator of the individual's genomic heterozygosity.

Zouros and Foltz (1986) argued that the assumption of random samples of loci being scored by the different biochemical techniques may not be appropriate. In fact, they argue that the empirical correlation most likely reflects the quantity

$$
\left[\sum_{i=1}^{L} H_{i} / \sum_{i=1}^{N} H_{i}\right]^{1 / 2},
$$

where $H_{i}$ is the heterozygosity at the $i$ th locus. In other words, the fraction of total heterozygosity at loci affecting the character accounted by the scored loci can be used as an indicator of how well the heterozygosity at the biochemical loci reflects the individual's genomic heterozygosity. It is true that in heterozygosity vs. morphological diversity studies only the highly polymorphic loci are employed. But Zouros and Foltz's assertion that electrophoretic techniques detect the polymorphisms with highest heterozygosities does not seem to be correct. Even if the sample of $L$ loci represents 
the most heterozygous loci of the genome, it can be shown that the correlation between an individual's measured heterozygosity (at $L$ loci) and the genomic heterozygosity is not given by expression (12), but by the expected value of

$$
\left[\sum_{i=1}^{L} H_{i}\left(1-H_{i}\right) / \sum_{i=1}^{N} H_{i}\left(1-H_{i}\right)\right]^{1 / 2} .
$$

There are two types of evidence suggesting that the expression (13) is not anywhere close to one.

First, in the statistical analysis of protein polymorphism in natural populations detected by electrophoresis, Fuerst et al. (1977) showed that the distribution of heterozygosity values across the studied electrophoretic loci agrees with the expected distribution under the assumption that these are random samples from the genome. Hence, the average heterozygosity at the population level is well estimated when a sufficient number of electrophoretic loci are used. This in turn suggests that the expected values of the individual terms in the numerator and denominator of expression (13) are roughly equal for the general electrophoretic surveys. If electrophoresis detected most of the genomic polymorphism, this would not have been the case. Hence, the assertion that most of the genomic variation is revealed by the surveyed loci does not seem to be correct.

Second, even though at present only a small number of studies have been made regarding the genetic diversity at the nucleotide level in natural populations of various organisms, the current estimate of nucleotide diversity is roughly of the order of $0.002-0.020$ on a per site basis (Kreitman, 1983; Chakravarti et al., 1984; Yager et al., 1984). However, since substitutions at the third position of a codon are roughly twice as frequent as those at the first or second positions (Nei, 1983), we can assume that the nucleotide diversity for the first or second position of the order $0.0015 \sim 0.015$, and that for the third position is $0.003 \sim 0.03$. Furthermore, if we assume that the probability of a random nucleotide change causing an electrophoretically detectable amino acid change is about 0.28 for the first position, one-third for the second position, and only one-twelfth for the third position (Kimura, 1983), the estimated heterozygosity per codon detectable by electrophoresis amounts to 0.0012 to 0.012 . On the other hand, in the general electrophoretic surveys the average heterozygosity in natural populations has been observed to be of the order of 0.47 or less (Nei and Graur, 1984). Assuming that an average protein has roughly 300 amino acids, the heterozygosity observed by elec- trophoresis per codon amounts to 0.0016 , which is close to the lower range of the estimate obtained from nucleotide diversity measures. We may therefore conclude that there is no solid evidence that expression (13) is much larger than $(L / N)^{1 / 2}$, as claimed by Zouros and Foltz (1987), and hence prediction of genomic heterozygosity from a survey of small number of highly polymorphic electrophoretic loci may not be appropriate.

\section{DISCUSSION}

The theory discussed above shows that: (a) the classification of individuals into different heterozygosity classes by phenotypes of a polygenic trait can be quite error prone, and (b) even if the underlying loci of polygenic traits are biochemically detectable, the negative relationship between heterozygosity of individuals and phenotypic variance, and a positive relationship between the frequency of modal class and individuals' heterozygosity can be explained by additive allelic effects of polygenic traits. Evidence is also provided that suggests that from the electrophoretic markers an individual's genomic heterozygosity may not be predicted accurately. Then, the question is how do we explain the observed relationship between phenotypic variance and frequency of phenotypic classes with electrophoretic heterozygosity without invoking interaction of loci that segregate independently of each other?

To explain these observations by the hypothesis of developmental homeostasis would require precise estimation of an individual's genomic heterozygosity by the surveyed loci. This is not the case, as discussed above. The other two popular hypotheses have been overdominant selection at the electrophoretic loci or selection at closely linked protein loci (associative overdominance). The later hypothesis would reflect that heterozygosity at the protein loci may have a synergistic effect with that at the loci governing the morphological trait. At this point, it might be mentioned that two sets of independently segregating loci may show correlated heterozygosity in individuals of a substructured population. Nei and Li (1973) showed that significant linkage disequilibrium might be produced at independently segregating loci in a substructured population. Sinnock (1975) showed that the two locus Wahlund effect results in a depletion of frequencies of double heterozygotes which can be more than the proportional decrease of heterozygotes at each individual locus. Thus, one may observe a correlation of 
heterozygosity levels at two unlinked loci for individuals in a subdivided population, and hence, the heterozygosity levels detected by protein loci may be correlated with heterozygosity of loci governing the morphologic trait to some extent, even if they are independently segregating. Of course, since the observed levels of linkage disequilibrium at the surveyed loci in natural populations is quite low (e.g., Mukai et al., 1971, 1974; Sinnock and Sing, 1972; Langley et al., 1974), it is unlikely that the correlation between heterozygosity levels at the biochemical loci and the loci governing morphologic variation can be explained by multi-locus Wahlund effects alone.

Evidence is now being accumulated suggesting that electrophoretically determined biochemical variation has measurable physiological consequences, some of which may modulate the phenotypic traits. Koehn et al. (1983) reviewed extensive experimental evidence of this suggestion. In humans, Orr et al. (1981) showed that variation in traits like serum cholesterol is affected by genotypes at the $\mathrm{ABO}$, Haptoglobin, Gamma globulin, and Secretor loci. Boerwinkle et al. (1986) showed that several biochemical traits indeed explain substantial proportions of genetic variability of many quantitative traits. At the DNA level too, restriction site polymorphisms are shown to explain significant component of variations of some physiological traits (e.g., Hanis et al., 1985). Thus, it is not totally unreasonable to assume that major genes residing at close linkage distance from some of the electrophoretically determined markers are the factors that at least partially control morphological variability. If this is the case, then the observation of an association of heterozygosity with phenotypic variability may truly be the reflection of the additive effects of the loci underlying these traits.

Several authors favour the hypothesis of overdominant selection to explain the association of phenotypic variance and biochemical heterozygosity (e.g., Mitton and Grant, 1984 and the cited references of their review). This hypothesis poses a number of problems regarding other facets of protein polymorphism data. For example, if overdominant selection operates on many protein loci, the average heterozygosity is expected to be much higher than the one predicted by the neutral mutation theory (Maruyama and Nei, 1981; Nei and Graur, 1984). But the observed levels of heterozygosities in various organisms are generally much smaller than their neutral expectations. If the neutral mutation model is in trouble to explain this (as suggested by Livshits and Kobyliansky,
1985), it would be more troublesome still to invoke the overdominant hypothesis as a general rule. Furthermore, in examining the allele frequency distributions of some 138 populations of various organisms, Chakraborty et al., (1980) showed that the allele frequency spectrum is generally $U$ shaped (which is in accordance with the neutral model), instead of being bell-shaped or W-shaped, which is the prediction of the overdominant model (Li, 1978). The observations on activity levels in heterozygotes and homozygotes of some enzymes are also against the overdominant hypothesis (see e.g., Harris, 1975; Kacser and Burns, 1981).

The associative overdominance hypothesis, as metioned earlier, may be an easier explanation for the correlation between the number of heterozygous loci and fitness related traits. This hypothesis apparently has its origin due to Jones (1917), as documented by Nei (1987). It is well known that in a finite population significant linkage disequilibrium between some traits may be produced by genetic drift (Hill and Robertson, 1968; Sved, 1968) and this would cause associative overdominance. Ohta's (1971) study also shows that when a protein locus with two alleles is linked with deleterious genes, heterozygotes at the protein locus may demonstrate higher fitness than homozygotes. Nevertheless, unless the loci governing the phenotypic traits are determined, these explanations cannot be tested directly.

Finally, it should be stated that not all reports of correlation between heterozygosity and fitness related traits are real. For example, Kobyliansky and Livshits (1985) claimed evidence of heterozygous advantages in human populations in terms of fertility in connection with five morphological characters: weight $(\mathrm{W})$, stature $(\mathrm{S})$, hand width (HAW), bigonial diameter (BIG), and ear width (EW). In showing these, they have classified 230 spouses from Mexican families into three classes (low $=1$, intermediate $=2$, and high $=3$ ), depending on their phenotypic scores.

Assuming that these traits are polygenic and heritable, they divided the parental data into nine mating types. On the supposition that the expected heterozygosity in the offspring of these mating can be arranged in ascending order, they reported the mean and s.d. of the number of living children from each mating type. Their analyses indicate that fertility, as determined by number of living children, is positively correlated with the heterozygosity when parental phenotypes as well as expected heterozygosity of the offspring are taken into account. From their data (summarised in table 1 of their paper), a simple analysis of variance may 
be conducted to see if the nine mating types show any significant differences in the fertility levels, the result of which is negative for each trait they examined, contrary to their findings. Furthermore, if we group the mating types by the expected heterozygosity of the offspring (which is error prone, as shown in this paper), the three groups of matings that give rise to expected heterozygosity of 0.0 (from matings $1 \times 1$, and $3 \times 3$ ), 0.5 (from matings $1 \times 2,2 \times 1,2 \times 3,3 \times 2$, and $2 \times 2$ ), and $1 \cdot 0$ (from matings $1 \times 3$ and $3 \times 1$ ) are also statistically homogeneous with regard to mean fertility.

Table 3 shows these computations from the data presented in Kobyliansky and Livshits (1985). These results are in direct contradiction with the assertion of their paper, but are in accordance with the findings of Chakraborty et al. (1986) that fertility is not affected by heterozygosity of the individuals determined from other loci, even though in the study of Chakraborty et al (1986) no effort is made to determine the expected heterozygosity of the offspring of mothers of different genotypes.

In conclusion, we may state that even when the observations on associations between biochemical heterozygosity and morphologic traits are real, they may not always be suggestive of selective factors involved in these traits. Any explanation invoking selection should, at the same time be compatible with other facets of protein polymorphism data, and this does not seem to be the case with an overdominant model. As shown here, the additive allelic effects at loci that are directly involved, or at disequilibrium with the trait may cause such association may be sufficient to explain some of these observations. In this regard the measured genotype approach (Boerwinkle et al., 1986) may help substantially to resolve the controversy regarding the underlying mechanism that might cause an association between phenotypic variability and biochemical heterozygosity.

Acknowledgements This work is supported by U.S. Public Health Service research grants from the National Institutes of Health and the National Science Foundation. Comments from Drs W. J. Schull, M. Nei, C. Stephens, and C. L. Hanis are greatly appreciated.

\section{REFERENCES}

BOERWINKLE, E., CHAKRABORTY, R. AND SING, C. F. 1986. The use of measured genotype information in the analysis of quantitative phenotypes in man. Ann. Hum. Genet., 50, $181-194$

CHAKRABORTY, R. 1981. The distribution of the number of heterozygous loci in an individual in natural populations. Genetics, 98, 461-466.

CHAKRABORTY, R., FERRELL, R. E., BARTON, S. A. AND SCHULL, W. J. 1986. Genetic polymorphism and fertility parameters in the Aymara of Chile and Bolivia. Ann. Hum. Genet., 50, 69-82.

CHAKRABORTY, R., FUERST, P. A. AND NEI, M. 1980. Statistical studies on protein polymorphism in natural populations. III. Distribution of allele frequencies and the number of alleles per locus. Genetics, 94, 1039-1063.

Table 3 Relationship between parental fitness and expected heterozygosity among children in a study from a Mexican population* Mating types of parents $\dagger$

\begin{tabular}{|c|c|c|c|c|c|c|c|c|c|c|c|}
\hline Trait $\ddagger$ & $n$ & $\begin{array}{l}\text { (a) } \\
\text { mean }\end{array}$ & s.d. & $n$ & $\begin{array}{l}\text { (b) } \\
\text { mean }\end{array}$ & s.d. & $n$ & $\begin{array}{l}(\mathrm{c}) \\
\text { mean }\end{array}$ & s.d. & $\begin{array}{l}\text { F-Ratio } \\
\text { df }\end{array}$ & $F$ \\
\hline w & 34 & $5 \cdot 20$ & $2 \cdot 21$ & 165 & $5 \cdot 86$ & $2 \cdot 41$ & 29 & $6 \cdot 21$ & $2 \cdot 13$ & $(2,225)$ & $1 \cdot 58$ \\
\hline HAW & 45 & $5 \cdot 25$ & 1.94 & 144 & $5 \cdot 93$ & $2 \cdot 44$ & 31 & $5 \cdot 81$ & $2 \cdot 57$ & $(2,217)$ & $1 \cdot 44$ \\
\hline BIG & 31 & $5 \cdot 65$ & $2 \cdot 25$ & 164 & $5 \cdot 66$ & $2 \cdot 25$ & 26 & 6.69 & $2 \cdot 47$ & $(2,218)$ & $2 \cdot 34$ \\
\hline EW & 23 & $5 \cdot 30$ & 1.99 & 178 & $5 \cdot 77$ & $2 \cdot 39$ & 19 & $6 \cdot 53$ & $2 \cdot 18$ & $(2,217)$ & $1 \cdot 45$ \\
\hline CI & 27 & $6 \cdot 18$ & $2 \cdot 84$ & 171 & $5 \cdot 85$ & $2 \cdot 33$ & 29 & $5 \cdot 33$ & $2 \cdot 06$ & $(2,224)$ & 0.94 \\
\hline
\end{tabular}

\footnotetext{
* Data taken from table 1 of Kobyliansky and Livshits (1985). The parental fitness is measured by the number of living children from each family.

† The mating types are classified by the phenotypic scores of each spouse: (1) with phenotypic score in the lowest 25 percentile; (2) with phenotypic score in the middle 25 to 75 percentile; and (3) with phenotypic score in the upper 25 percentile of the distributions. The three mating types are: (a) $1 \times 1$ and $3 \times 3$ (the matings for which the expected heterozygosity of the offspring is 0 ); (b) $1 \times 2,2 \times 1,2 \times 3,3 \times 2$, and $2 \times 2$ (the matings for which the expected heterozygosity in the offspring is 0.5 ); and (c) $1 \times 3$ and $3 \times 1$ (the matings for which the expected heterozygosity in the offspring is 1 ).

\$ The traits are: W (weight); S (stature); HAW (Hand width); BIG (Bigonial diameter); EW (ear width); EH (ear height); MN (menton-nasion index); and CI (cephalic index).
} 
CHAKRABORTY, R. AND RYMAN, N. 1983. Relationship between mean and variance of genotypic values with heterozygosity per individual in a natural population. Genetics, 103, 149-152.

CHAKRAVARTI, A., BUETOW, K. H., ANTONARAKIS, S. E., WABER, P. G. AND BOEHM, C. D., KAZAZIAN, H. H. 1984. Nonuniform recombination within the human $\beta$-globin gene cluster. Amer. J. Hum. Genet., 36, 1239-1258.

FELLER, w. 1950. An Introduction to Probability Theory and its Applications. Volume 1, John Wiley, New York.

FUERST, P. A., CHAK RABORTY, R. AND NEI, M. 1977. Statistical studies on protein polymorphism in natural populations I. Distribution of single locus heterozygosity. Genetics, 86 , $455-483$.

GOTTLIEB, L. D. 1977. Genotypic similarity of large and small individuals in a natural population of the annual plant Stephanomeria exigua ssp. coronaria (compositae). Jour. Ecol., 65, 127-134.

HANDFORD, P. 1980. Heterozygosity at enzyme loci and morphological variation. Nature, 286, 261-262.

HANIS, C. L., CHAKRABORTY, R. AND HEWETT-EMMETT, D. 1985. How much of variability in apolipoprotein AII concentrations is explained by polymorphism adjacent to ApoII gene? Lancet, $i, 1339-1340$.

HARR IS, H. 1975. The Principles of Human Biochemical Genetics, North-Holland, New York.

HILL, W. G. AND ROBERTSON, A. 1968. Linkage disequilibrium in finite populations. Theor. Appl. Genet., 38, 226-231.

JONES, D. F. 1917. Dominance of linked factors as a means of accounting for heterosis. Genetics, 2, 466-479.

KACSER, H. AND BURNS, J. A. 1981. The molecular basis of dominance. Genetics, 97, 639-666.

KIMURA, M. 1983. Rare variant alleles in the light of the neutral theory. Mol. Biol. Evol., 1, 84-93.

KNOWLES, P. AND GRANT. M. C. 1981. Genetic patterns associated with growth variability in ponderosa pine. Amer. Jour. Bot., 68, 942-946.

KNOWLES, P. AND MITTON, J. B. 1980. Genetic heterozygosity and radial growth variability in Pinus contorta. Silvae Genetica, 29, 114-118.

KOEHN, R. K., ZERA, A. J. AND HALL, J. G. 1983. Enzyme polymorphism and natural selection. In $\mathrm{Nei}, \mathrm{M}$. and Koehn, R. K. (eds.) Evolution of Genes and Proteins, Sinauer, Sunderland, pp. 115-137.

KOBYLIANSKY, E. ANI) L.IVSHITS, G. 1985. Differential fertility and morphological constitution of spouses. Z. Morph. Anthrop., 76, 95-105.

KREITMAN, M. 1983. Nucleotide polymorphism at the alcohol dehydrogenase locus of Drosophila melanogaster. Nature, 304, 412-417.

LANGLEY, C. H., TOBARI, Y. N. AND KOJIMA, K. 1974. Linkage disequilibrium in natural populations of Drosophila melanogaster. Seasonal variation. Genetics, 86, 447-454.

LI, W-H. 1978. Maintenance of genetic variability under the joint effect of mutation, selection, and random drift. Genetics, 90, 349-382.

LIVSHITS, G. AND KOBYLIANSKY, E. 1984. Biochemical heterozygosity as a predictor of developmental homeostasis in man. Ann. Hum. Genet., 48, 173-184.

LIVSHITS, G. AND KOBYLIANSKY, E. 1985. Lerner's concept of developmental homeostasis and the problem of heterozygosity level in natural populations. Heredity, 55, 341-353.

MARUYANA, T. AND NEI, M. 1981. Genetic variability maintained by mutation and overdominant selection in finite populations. Genetics, 98, 441-459.

MITTON, J. B. AND GRANT, M. C. 1984. Association among heterozygosity, growth rate and developmental homeostasis. Ann. Rev. Ecol. Syst., 15, 479-499.

MITTON, J. B., KNOWLES, P., STURGEON, K. B., LINHART, Y. B. AND DAVIS, M. 1981. Associations between heterozygosity and growth rate variables in three western forest trees. In Conkle, M. T. (ed.) Isozymes of North American Forest Trees and Forest Insects. Gen. Tech. Rep. PSW-48, USDA Forest Service, Pacific Southwest Forest Range Experimental Station.

MITTON, J. B. AND PIERCE, B. A. 1980. The distribution of individual heterozygosity in natural populations. Genetics, $95,1043-1054$

MUKAI, T., METTLER, L. E. AND CHIGUSA, S. I. 1971. Linkage disequilibrium in a local population of Drosophila melanogaster. Proc. Natl. Acad. Sci. USA, 68, 1056-1069.

MUKAI, T., WATANABE, T. K. AND YAMAgUCHI, O. 1974. The genetic structure of natural populations of Drosophila melanogaster. XII. Linkage disequilibrium in a large local population. Genetics, 77, 771-793.

NEI, M. 1983. Genetic polymorphism and the role of mutation in evolution. In Nei, M. and Koehn, R. K. (eds.) Evolution of Genes and Proteins, Sinauer, Sunderland, pp. 165-190.

NEI, M. 1987. Molecular Evolutionary Genetics, Columbia Univ. Press. New York.

NEI, M. AND GRAUER, D. 1984. Extent of protein polymorphism and the neutral mutation theory. Evol. Biol., 17, 73-118.

NEI, M. AND LI, W-H. 1973. Linkage disequilibrium in subdivided populations. Genetics, 75, 213-219.

OHTA, T. 1971. Associative overdominance caused by linked detrimental mutations. Genet. Res., 18, 277-286.

ORR, J. D., SING, C. F. AND MOLL, P. P. 1981. Analysis of genetic and environmental sources of variation in serum cholesterol in Tecumseh, Michigan. V1. A search for genotype by environment interaction. Jour Chron. Dis., 34, 545-559.

PIERCE, B. A. AND MITTON, J. B. 1982. Allozyme heterozygosity and growth in tiger salamander, Ambyostoma tigrinum. Jour. Hered, 73, 250-253.

SINNOCK, P. 1975. The Wahlund effect for the two-loci model. Am. Nat., 109, 565-570.

SINNOCK, P. AND SING, C. F. 1972. Analysis of multilocus genetic systems in Tecumseh, Michigan. II. Consideration of the correlation between nonalleles in gametes. Amer. J. Hum. Genet., 24, 393-415.

SMOUSE, P. E. 1986. The fitness consequences of multi-loçus heterozygosity under the multiplicative overdominance and inbreeding depression models. Evol. 40, 946-957.

SVED, J. A. 1968. The stability of linked systems of loci with a small population size. Genetics, 59, 543-563.

YAGER, L. N., KAUMEYER, J. F. AND WEINBERG, E. S. 1984. Evolving sea urchin histone genes-nucleotide polymorphisms in the $\mathrm{H} 4$ gene and spacers of Stronglocentrotus purpuratus. Jour. Mol. Evol., 20, 215-226.

ZOUROS, E. AND FOLTZ, D. W. 1987. The use of allelic isozyme variation for the study of heterosis. Isozymes (In press). 